\title{
SOME GENERAL LOCAL VARIATIONAL PRINCIPLES
}

\author{
PAUL BINDING AND QIANG YE
}

(Communicated by Palle E. T. Jorgensen)

\begin{abstract}
Local variational min-sup characterizations are presented for the real spectrum of a selfadjoint operator pencil. Instead of minimizing over all subspaces of fixed codimension as in the classical result, the new characterizations minimize over subspaces that are close to extremal subspaces. In this way, the entire real spectrum, including continuous spectrum, can be characterized.
\end{abstract}

\section{INTRODUCTION}

For a selfadjoint operator that is bounded below, there is a classical minimax theorem that characterizes the discrete end of the spectrum. Over the years, this minimax characterization has played a very important role in the investigation of spectra of selfadjoint operators. It is natural to ask whether similar characterizations exist for the entire spectrum of a selfadjoint operator, or more generally for the real spectrum of a selfadjoint operator pencil $\lambda A-B$. In recent works [AM, NY], a minimax theorem of the classical form was established for certain eigenvalues of a selfadjoint matrix (or operator) pencil. In this paper it is our intention to consider a minimax characterization that has a weaker form but applies to all the real spectrum.

We first note that the minimax principle is closely related to the stationary property of the Rayleigh quotient, which is a local result. It turns out that this is an appropriate framework for obtaining a general minimax theorem. We present a local minimax characterization for the real spectrum of a selfadjoint operator pencil. One extremum in our theorem is taken over subspaces that are close to an extremal subspace instead of over all subspaces of specific codimension as in the classical case. It is in this sense that we regard it as a local characterization. In the rest of this section, we present the basic theorem and some direct implications. The proofs are in $\S 3$, and a more complicated application is given in $\S 4$.

Let $H$ be a separable Hilbert space. We first introduce a neighborhood system in the set of closed subspaces. Given such a subspace $S_{0}$, we choose an orthonormal basis $\mathscr{B}$ for it, and we consider subspaces spanned by elements

Received by the editors June 6, 1990.

1980 Mathematics Subject Classification (1985 Revision). Primary 49G05.

Key words and phrases. Minimax principle, selfadjoint pencils.

The first author's research was supported by a Canadian NSERC Operating Grant.

The second author's research was supported by a University of Calgary Research Fellowship. 
that are slightly perturbed from $\mathscr{B}$. Specifically, we introduce the following definition.

Definition 1.1. Let $S_{0}$ and $T_{0}$ be two closed subspaces such that $H=S_{0}+T_{0}$. Then for $\varepsilon>0$,

$$
N_{\varepsilon}\left(S_{0}, T_{0}\right)=\left\{\operatorname{span}\left\{e_{n}+e_{n}^{\prime}\right\}_{n=0}^{+\infty}: e_{n}^{\prime}=\sum_{m=-\infty}^{+\infty} \varepsilon_{m n} e_{m}, \sum_{\substack{m=-\infty \\ n=0}}^{+\infty}\left|\varepsilon_{m n}\right|^{2}<\varepsilon\right\}
$$

is called an $\varepsilon$-neighborhood of $S_{0}$ relative to $T_{0}$, where $\left\{e_{n}\right\}_{n=0}^{+\infty}$ and $\left\{e_{n}\right\}_{-n=1}^{+\infty}$ are orthonormal bases of $S_{0}$ and $T_{0}$ respectively.

We show in $\S 2$ that the definition of $N_{\varepsilon}\left(S_{0}, T_{0}\right)$ is independent of the choices of orthonormal bases $\left\{e_{n}\right\}_{n=0}^{\infty}$ and $\left\{e_{n}\right\}_{-n=1}^{+\infty}$ and thus pertains only to the subspaces $S_{0}$ and $T_{0}$.

Let $A, B$ be two bounded selfadjoint operators on $H$, and let

$$
C_{ \pm}=\{x \in H: \pm(A x, x)>0\} \text {. }
$$

Our starting point is the following basic pair of assumptions for some fixed $\lambda \in \mathbb{R}$.

(1) $H$ admits the direct sum decomposition $H=S_{\lambda}+T_{\lambda}$ with $S_{\lambda}, T_{\lambda}$ closed and

$$
\lambda=\sup _{x \in S_{\lambda} \cap C_{+}}(B x, x) /(A x, x) .
$$

(2) There is a fixed $\delta>0$, so that for any $\gamma>0$, there exist a subspace $T_{\gamma} \supset T_{\lambda}$ and an element $x_{\gamma} \in T_{\gamma} \cap S_{\lambda}$ satisfying $\left(A x_{\gamma}, x_{\gamma}\right)>\delta\left\|x_{\gamma}\right\|^{2}$ and

$$
\inf _{x \in T_{\gamma} \cap C_{+}}(B x, x) /(A x, x) \geq \lambda-\gamma .
$$

We now state the local minimax theorem.

Theorem 1.2. Let $A, B$ be two bounded selfadjoint operators on a separable Hilbert space $H$. If assumptions (1) and (2) hold for some $\lambda \in \mathbb{R}$, then there is $\varepsilon>0$ such that

$$
\lambda=\min _{S \in N_{\varepsilon}\left(S_{\lambda}, T_{\lambda}\right)} \sup _{x \in S \cap C_{+}}(B x, x) /(A x, x) .
$$

To apply this abstract theorem to concrete problems, we need to construct $S_{\lambda}, T_{\lambda}$, and $T_{\gamma}$ so that assumptions (1) and (2) are satisfied. Here we present two direct applications. If $E$ is the resolution of the identity for a selfadjoint operator $C$ on $H$, so $C x=\int_{-\infty}^{\infty} \lambda d E(\lambda) x$ for all $x \in H$, then we write

$$
\left.\left.E_{\lambda}=E(]-\infty, \lambda\right]\right) \text {. }
$$

Theorem 1.3. Let $E$ be the resolution of the identity for a bounded selfadjoint operator $B$ on $H$. If

$$
\left.\left.E(] \lambda_{0}-\gamma, \lambda_{0}\right]\right) \neq 0 \text { for all } \gamma>0
$$

(so $\lambda_{0}$ is an eigenvalue of $B$ or a point of continuous spectrum not on the left boundary), then there is $\varepsilon>0$, such that

$$
\lambda_{0}=\min _{S \in N_{\varepsilon}\left(S_{0}, T_{0}\right)} \sup _{x \in S, x \neq 0}(B x, x) /(x, x),
$$


where $S_{0}=\mathfrak{R}\left(E_{\lambda_{0}}\right), T_{0}=\mathfrak{R}\left(I-E_{\lambda_{0}}\right)$ (see (1.2)).

We say that $\lambda_{0}$ is an eigenvalue of the operator pencil $P: \lambda \rightarrow \lambda A-B$ if $B x=\lambda_{0} A x$ for some nonzero $x \in H$, i.e. 0 is an eigenvalue of $\lambda_{0} A-B$. Also $\lambda_{0}$ is $A$-positive (resp. $A$-negative) if $x \in C_{+}$(resp. $x \in C_{-}$) of (1.1).

Theorem 1.4. If $\lambda_{0}$ is an A-positive eigenvalue of $P$, let $E$ be the resolution of the identity for $\lambda_{0} A-B$ and let $S_{0}=\mathfrak{R}\left(E_{0}\right), T_{0}=\mathfrak{R}\left(I-E_{0}\right)$. Then there is $\varepsilon>0$ such that

$$
\lambda_{0}=\min _{S \in N_{\varepsilon}\left(S_{0}, T_{0}\right)} \sup _{x \in S \cap C_{+}}(B x, x) /(A x, x) .
$$

Our final application is Theorem 4.2, which extends Theorem 1.3 to Pontryagin space and Theorem 1.4 to include continuous spectrum in an appropriate sense. Both extensions are, however, at the cost of extra conditions on the pair $(A, B)$.

All the results in this paper have been restricted to min-sup characterizations and $A$-positive eigenvalues. There are dual characterizations of max-inf type, and also of $A$-negative eigenvalues, which can be obtained similarly and are left to the reader.

\section{Preliminaries}

In this section, we prove an essential lemma on intersection of subspaces. First, however, we clarify Definition 1.1 .

Theorem 2.1. The definition of $N_{\varepsilon}\left(S_{0}, T_{0}\right)$ is independent of the choice of orthonormal bases for $S_{0}$ and $T_{0}$.

Proof. If $S_{0}=\operatorname{span}\left\{f_{n}\right\}_{n=0}^{+\infty}, T_{0}=\operatorname{span}\left\{f_{n}\right\}_{-n=1}^{+\infty}$, where $\left\{f_{n}\right\}_{n=0}^{+\infty}$ and $\left\{f_{n}\right\}_{-n=1}^{+\infty}$ are orthonormal respectively, let

$$
e_{n}=\sum_{l=0}^{+\infty} \alpha_{n l} f_{l} \quad \text { for } n \geq 0, \quad e_{n}=\sum_{-l=1}^{+\infty} \alpha_{n l} f_{l} \text { for } n \leq-1
$$

and

$$
f_{l}=\sum_{n=0}^{+\infty} \beta_{l n} e_{n} \quad \text { for } l \geq 0, \quad f_{l}=\sum_{-n=1}^{+\infty} \beta_{l n} e_{n} \quad \text { for } l \leq-1
$$

Then

$$
\begin{array}{ll}
\sum_{l=0}^{+\infty} \beta_{n l} \alpha_{l m}=\sum_{l=0}^{+\infty} \alpha_{n l} \beta_{l m}=\delta_{n m} & \text { for } m, n \geq 0, \\
\sum_{-l=1}^{+\infty} \beta_{m l} \alpha_{l n}=\sum_{-l=1}^{+\infty} \alpha_{n l} \beta_{l m}=\delta_{n m} & \text { for } m, n \leq-1,
\end{array}
$$

and

$$
\sum_{n=0}^{+\infty} \beta_{l n} \bar{\beta}_{k n}=\delta_{l k} \text { for } l, k \geq 0
$$


Now, for a subspace $S=\operatorname{span}\left\{e_{n}+e_{n}^{\prime}\right\}_{n=0}^{+\infty}$ with $e_{n}^{\prime}=\sum_{m=-\infty}^{+\infty} \varepsilon_{m n} e_{m}$ and $\sum_{m=-\infty, n=0}^{+\infty}\left|\varepsilon_{m n}\right|^{2}<\varepsilon$, by $(2.1)$ we have

$$
S=\operatorname{span}\left\{\sum_{n=0}^{+\infty} \beta_{l n}\left(e_{n}+e_{n}^{\prime}\right)\right\}_{l=0}^{+\infty}=\operatorname{span}\left\{f_{l}+\sum_{k=-\infty}^{+\infty} \varepsilon_{k l}^{\prime} f_{k}\right\}_{l=0}^{+\infty},
$$

where

$$
\varepsilon_{k l}^{\prime}=\sum_{n=0}^{+\infty} \beta_{l n} \sum_{-m=1}^{+\infty} \varepsilon_{m n} \alpha_{m k} \quad \text { for } k \leq-1 \text { and } l \geq 0
$$

and

$$
\varepsilon_{k l}^{\prime}=\sum_{n=0}^{+\infty} \beta_{l n} \sum_{m=0}^{+\infty} \varepsilon_{m n} \alpha_{m k} \quad \text { for } k \geq 0 \text { and } l \geq 0 .
$$

Furthermore, by (2.2) and (2.3), we have $\alpha_{j k}=\bar{\beta}_{k j}$. Then after a computation, we reach

$$
\sum_{\substack{k=-\infty \\ l=0}}^{+\infty}\left|\varepsilon_{k l}^{\prime}\right|^{2}=\sum_{\substack{m=-\infty \\ n=0}}^{\infty}\left|\varepsilon_{m n}\right|^{2}
$$

The proof is complete.

A basic problem in the construction of minimax principles is whether two given subspaces intersect nontrivially. In the classical case, this intersection can be deduced from dimension arguments. In the general case, both subspaces in question could be of infinite dimension. We show that given two nontrivially intersecting subspaces, they still intersect if one of the subspaces is perturbed slightly in the sense of Definition 1.1.

Lemma 2.2. Let $A$ be a bounded selfadjoint operator on a separable Hilbert space $H=S_{0}+T_{0}$ with $S_{0}, T_{0}$ closed. Given $\delta>0$, there is $\varepsilon>0$, such that if $S \in N_{\varepsilon}\left(S_{0}, T_{0}\right), T_{0} \subset T$, and $x_{T} \in S_{0} \cap T$ with $\left(A x_{T}, x_{T}\right)>\delta\left\|x_{T}\right\|^{2}$, then there is $x \in S \cap T$ with $(A x, x)>0$.

Proof. Let $S_{0}=\operatorname{span}\left\{e_{n}\right\}_{n=0}^{\infty}, T_{0}=\operatorname{span}\left\{e_{n}\right\}_{-n=1}^{+\infty}$, and $S=\operatorname{span}\left\{e_{n}+e_{n}^{\prime}\right\}_{n=0}^{+\infty}$ with $e_{n}^{\prime}=\sum_{m=-\infty}^{+\infty} \varepsilon_{m n} e_{m}$ and $\sum_{m=-\infty}^{+\infty} n=0\left|\varepsilon_{m n}\right|^{2}<\varepsilon<1$. For $x_{T} \in S_{0} \cap T$, we can write $x_{T}=\sum_{n=0}^{+\infty} \beta_{n} e_{n}$. We then consider the following equation in $\alpha_{n}$ :

$$
\alpha_{n}+\sum_{m=0}^{+\infty} \alpha_{m} \varepsilon_{n m}=\beta_{n}
$$

for $n=0,1,2, \ldots$ Equivalently, this can be written in infinite matrix form

$$
(I+E) \alpha=\beta
$$

on $l_{2}$, where

$$
E=\left[\begin{array}{cccc}
\varepsilon_{00} & \varepsilon_{01} & \varepsilon_{02} & \cdots \\
\varepsilon_{10} & \varepsilon_{11} & \varepsilon_{12} & \cdots \\
\vdots & \vdots & \vdots &
\end{array}\right], \quad a=\left[\begin{array}{c}
\alpha_{0} \\
\alpha_{1} \\
\alpha_{2} \\
\vdots
\end{array}\right], \quad \text { and } \quad \beta=\left[\begin{array}{c}
\beta_{0} \\
\beta_{1} \\
\beta_{2} \\
\vdots
\end{array}\right]
$$


Since $\|E\|_{2} \leq \sum_{m=0, n=0}^{+\infty}\left|\varepsilon_{m n}\right|^{2}<\varepsilon<1$, (2.5) has the unique solution $\alpha=$ $(I+E)^{-1} \beta$. Moreover, $\|\alpha\|_{2} \leq\|\beta\|_{2} /\left(1-\|E\|_{2}\right) \leq\left\|x_{T}\right\|_{2} /(1-\varepsilon)$. We now let

$$
x=\sum_{n=0}^{+\infty} \alpha_{n}\left(e_{n}+e_{n}^{\prime}\right) \in S .
$$

Then by (2.4), we have $x=y_{0}+x_{T}$, where

$$
y_{0}=\sum_{-m=1}^{+\infty}\left(\sum_{n=0}^{+\infty} \varepsilon_{m n} \alpha_{n}\right) e_{m} \in T_{0} \subset T,
$$

so $x \in S \cap T$. Furthermore

$$
\left\|y_{0}\right\|^{2}=\sum_{-m=1}^{+\infty}\left|\sum_{n=0}^{+\infty} \varepsilon_{m n} \alpha_{n}\right|^{2} \leq\left(\sum_{-m=1}^{+\infty} \sum_{n=0}^{+\infty}\left|\varepsilon_{m n}\right|^{2}\right)\left(\sum_{n=0}^{+\infty}\left|\alpha_{n}\right|^{2}\right)<\frac{\varepsilon\left\|x_{T}\right\|^{2}}{(1-\varepsilon)^{2}}
$$

and

$$
\begin{aligned}
(A x, x) & =\left(A x_{T}, x_{T}\right)+2 \operatorname{Re}\left(A x_{T}, y_{0}\right)+\left(A y_{0}, y_{0}\right) \\
& >\delta\left\|x_{T}\right\|^{2}-2\left\|x_{T}\right\| \cdot\|A\| \cdot\left\|y_{0}\right\|-\|A\|\left\|y_{0}\right\|^{2} \\
& \geq\left(\delta-\frac{2 \sqrt{\varepsilon}}{1-\varepsilon}\|A\|-\frac{\varepsilon}{(1-\varepsilon)^{2}}\|A\|\right) \cdot\left\|x_{T}\right\|^{2} .
\end{aligned}
$$

Thus, choosing $\varepsilon$ small enough to ensure $\delta-\left(2 \sqrt{\varepsilon} /(1-\varepsilon)+\varepsilon /(1-\varepsilon)^{2}\right)\|A\|>0$, we have $(x, A x)>0$. This completes the proof.

\section{Proofs of the MAIN THEOREMS}

Proof of Theorem 1.2. Choose $\delta$ as in assumption (2). Thus for any $\gamma>0$, there is a subspace $T_{\gamma} \supset T_{\lambda}$ and $x_{\gamma} \in T_{\gamma} \cap S_{\lambda}$ such that $\left(A x_{\gamma}, x_{\gamma}\right)>\delta\left\|x_{\gamma}\right\|^{2}$ and

$$
\inf _{x \in T_{\gamma} \cap C_{+}}(B x, x) /(A x, x) \geq \lambda-\gamma .
$$

Now let $\varepsilon$ correspond to $\delta$ in Lemma 2.2. Then for given $S \in N_{\varepsilon}\left(S_{\lambda}, T_{\lambda}\right)$, there is $x_{0} \in S \cap T_{\gamma} \cap C_{+}$. Thus

$$
\sup _{x \in S \cap C_{+}} \frac{(B x, x)}{(A x, x)} \geq \frac{\left(B x_{0}, x_{0}\right)}{\left(A x_{0}, x_{0}\right)} \geq \inf _{x \in T_{\gamma} \cap C_{+}} \frac{(B x, x)}{(A x, x)} \geq \lambda-\gamma .
$$

Noting that $\gamma$ is any positive number, we have

$$
\sup _{x \in S \cap C_{+}}(B x, x) /(A x, x) \geq \lambda .
$$

This together with assumption (1) proves the theorem.

As pointed out in $\S 1$, several applications can be derived from Theorem 1.2. We now proceed to the proofs of the remaining theorems.

Proof of Theorem 1.3. It is enough to check assumptions (1) and (2) with $A=I$. For $x \in S_{0}=\mathfrak{R}\left(E_{\lambda_{0}}\right)$

$$
(B x, x)=\int_{-\infty}^{\lambda_{0}} \lambda(d E(\lambda) x, x) \leq \lambda_{0}(x, x) .
$$


In view of (1.3),

$$
\sup _{x \in S_{0}, x \neq 0}(B x, x) /(x, x)=\lambda_{0}
$$

and we have verified assumption (1). Now, for any $\gamma>0$, let $T_{\gamma}=\mathfrak{R}\left(I-E_{\lambda_{0}-\gamma}\right)$. Then $T_{\gamma} \supset T_{0}$. Furthermore, $S_{0} \cap T_{\gamma}=\mathfrak{R}\left(E_{\lambda_{0}}-E_{\lambda_{0}-\gamma}\right) \neq\{0\}$ so $x_{\gamma}$ exists with $\delta=1 / 2$, and

$$
\inf _{x \in T_{\gamma}, x \neq 0} \frac{(B x, x)}{(x, x)} \geq \lambda_{0}-\gamma .
$$

Hence, assumption (2) is also satisfied.

Proof of Theorem 1.4. Again, we only need to check assumptions (1) and (2). Let $x_{0}$ be an eigenvector corresponding to $\lambda_{0}$ with $\left(A x_{0}, x_{0}\right)>0$. For $x \in$ $S_{0}=\mathfrak{R}\left(E_{0}\right)$,

$$
\left(\left(B-\lambda_{0} A\right) x, x\right) \leq 0
$$

i.e.

$$
(B x, x) \leq \lambda_{0}(A x, x) \text {. }
$$

Since $x_{0} \in S_{0} \cap C_{+}$, we have

$$
\sup _{x \in S_{0} \cap C_{+}}(B x, x) /(A x, x)=\lambda_{0} .
$$

Now, for any $\gamma>0$, let $T_{\gamma}=\mathfrak{R}(I-E(]-\infty, 0[))$. Then $T_{\gamma} \supset T_{0}$ and $x_{0} \in$ $S_{0} \cap T_{\gamma}$. Obviously $\left(A x_{0}, x_{0}\right)>\delta\left(x_{0}, x_{0}\right)$ with $\delta=\left(A x_{0}, x_{0}\right) / 2\left(x_{0}, x_{0}\right)>0$. Also, for $x \in T_{\gamma}$,

$$
\left(\left(B-\lambda_{0} A\right) x, x\right) \geq 0 .
$$

Thus

$$
\inf _{x \in T_{y} \cap C_{+}}(B x, x) /(A x, x) \geq \lambda_{0}>\lambda_{0}-\gamma .
$$

Hence both assumptions (1) and (2) are satisfied.

\section{A Variational PRINCIPLe in Pontryagin SPace}

The basis for this section is a direct sum of selfadjoint pencils $P_{1} \oplus P_{2}$ on $H=H_{1} \oplus H_{2}$ where $\operatorname{dim} H_{1}$ is finite, Theorem 1.4 applies to $P_{1}$ and Theorem 1.3 applies to $P_{2}$. Specifically, we write

$$
P_{1}(\lambda)=\lambda A_{1}-B_{1}, \quad P_{2}(\lambda)=\lambda I_{2}-B_{2},
$$

where $A_{1}$ is nonsingular. We remark that a variety of pencils on a given Hilbert space $H$ can be transformed into such direct sums [B]. Evidently, the operator

$$
Q=A^{-1}\left(B_{1} \oplus B_{2}\right)
$$

is selfadjoint in the Pontryagin space $H_{A}$ defined as $H$ but with the inner product generated by $A=A_{1} \oplus I_{2}$. (Note that the Hilbert majorant topology of $H_{A}$ is just that of $H$.) Conversely, any selfadjoint operator $L$ on a Pontryagin space admits such a direct sum decomposition, provided a certain nondegeneracy condition holds [B, p. 337] or equivalently provided there are no singular critical points [L, p. 40]. As a simple example, if $L$ is compact then the above conditions hold if $N(L)$ is nondegenerate (e.g. if $L$ is $1-1$ ) (cf. [BS, Theorem 1]).

We have the following result for $P_{1}$. 
Lemma 4.1. For any $\lambda \in \mathbb{R}$, there exist complementary subspaces $S_{\lambda}^{1}$ and $T_{\lambda}^{1}$ of $H_{1}$, such that

$$
\sup _{x \in S_{\lambda}^{1} \cap C_{+}} \frac{\left(B_{1} x, x\right)}{\left(A_{1} x, x\right)} \leq \lambda \leq \inf _{x \in T_{\lambda}^{1} \cap C_{+}} \frac{\left(B_{1} x, x\right)}{\left(A_{1} x, x\right)} .
$$

Furthermore, if $\lambda$ is an A-positive eigenvalue of $P_{1}$ with a corresponding eigenvector $x_{0} \in C_{+}$, then $x_{0} \in S_{\lambda}^{1}$.

A construction of $S_{\lambda}^{1}, T_{\lambda}^{1}$ for certain eigenvalues $\lambda$ is contained in the proof of Theorem 3.1 of [NY] in the context of matrix pencils, but in fact this construction applies to any real number and not just the eigenvalues. The details are omitted here, but we remark that these subspaces $S_{\lambda}^{1}$ and $T_{\lambda}^{1}$ involve splitting spectral subspaces for $A_{1}^{-1} B_{1}$ in a fashion that is in general not unique.

If $E$ is the resolution of the identity for $B_{2}$, then we define

$$
S_{\lambda}=S_{\lambda}^{1}+\mathfrak{R}\left(E_{\lambda}\right) \text { and } T_{\lambda}=T_{\lambda}^{1}+\mathfrak{R}\left(I-E_{\lambda}\right),
$$

where $S_{\lambda}^{1}, T_{\lambda}^{1}$ are obtained from Lemma 4.1, and $E_{\lambda}$ comes from (1.2).

Theorem 4.2. Let $P_{1}, P_{2}$, and $Q$ be as in (4.1), (4.2). If $\lambda$ is an A-positive eigenvalue or a point of continuous spectrum of $Q$ (not on the left boundary), then there is $\varepsilon>0$ such that

$$
\lambda=\min _{S \in N_{\varepsilon}\left(S_{\lambda}, T_{\lambda}\right)} \sup _{x \in S \cap C_{+}}(B x, x) /(A x, x),
$$

where $S_{\lambda}, T_{\lambda}$ come from (4.3) and $N_{\varepsilon}$ refers to the topology of $H_{A}$, i.e., of $H$. Proof. Again, we check assumptions (1) and (2).

(1) For any $x \in S_{\lambda}$, we write $x=x_{1}+x_{2}$ with $x_{1} \in S_{\lambda}^{1}$ and $x_{2} \in \mathfrak{R}\left(E_{\lambda}\right)$, so

$$
(B x, x)=\left(B x_{1}, x_{1}\right)+\left(B x_{2}, x_{2}\right) \leq \lambda\left(A x_{1}, x_{1}\right)+\lambda\left(A x_{2}, x_{2}\right)=\lambda(A x, x) \text {. }
$$

Furthermore, if $\lambda$ is an $A$-positive eigenvalue with a corresponding eigenvector $x_{0} \in C_{+}$, then we can assume without loss of generality that either $x_{0} \in H_{1}$ or $x_{0} \in H_{2}$. Thus either $x_{0} \in S_{\lambda}^{1}$ or $x_{0} \in \mathfrak{R}\left(E_{\lambda}\right)$, i.e., $x_{0} \in S_{\lambda} \cap C_{+}$whence

$$
\lambda=\sup _{x \in S_{\lambda} \cap C_{+}}(B x, x) /(A x, x) \text {. }
$$

If $\lambda \in \sigma_{c}(Q)$ but not on the left boundary, then $\lambda \in \sigma_{c}\left(B_{2}\right)$ and (1.3) holds. Arguing as for (3.1), we obtain

$$
\lambda=\sup _{x \in R\left(E_{\lambda}\right) \cap C_{+}}(B x, x) /(A x, x),
$$

which leads to (4.4) again.

(2) If $\lambda$ is an $A$-positive eigenvalue, there is an eigenvector $x_{0} \in S_{\lambda} \cap C_{+}$as in (1). Let $T_{\gamma}=T_{\lambda}+\operatorname{span}\left\{x_{0}\right\}$ for any $\gamma>0$. Then $x_{0} \in S_{\lambda} \cap T_{\gamma}$ and $T_{\gamma} \supset T_{\lambda}$. Further

$$
\inf _{x \in T_{\gamma} \cap C_{+}}(B x, x) /(A x, x)=\lambda>\lambda-\gamma .
$$

If $\lambda \in \sigma_{c}(Q)$ but not on the left boundary, let $T_{\gamma}=T_{\lambda}^{1}+\Re\left(I-E_{\lambda-\gamma}\right)$ for any $\gamma>0$. Then $S_{\lambda} \cap T_{\gamma} \cap C_{+}=\mathfrak{R}\left(E_{\lambda}-E_{\lambda-\gamma}\right) \subset H_{2}$ is nontrivial, so $x_{\gamma}$ exists with $\delta=1 / 2$. Further

$$
\inf _{x \in T_{\gamma} \cap C_{+}}(B x, x) /(A x, x) \geq \lambda_{0}-\gamma
$$

so, assumption (2) is also satisfied. 


\section{REFERENCES}

[AM] W. Allegretto and A. B. Mingarelli, Boundary problems of the second order with an indefinite weight function, J. Reine Angew. Math. 398 (1989), 1-24.

[B] P. A. Binding, A canonical form for self-adjoint pencils in Hilbert space, Integal Equations Operator Theory, 12 (1989), 324-342.

[BS] P. A. Binding and K. Seddighi, On root vectors of self-adjoint pencils, J. Funct. Anal. 70 (1987), 117-125.

[L] H. Langer, Spectral functions of definitizable operators in Krein space, Functional Analysis, Proceedings, Dubrovnik 1981, Lecture Notes in Math., vol. 948, Springer-Verlag, New York, 1981, pp. 1-46.

[NY] B. Najman and Q. Ye, A minimax characterization for eigenvalues of hermitian pencils, Linear Algebra Appl. 144 (1991), 217-230.

Department of Mathematics and Statistics, University of Calgary, Calgary, Alberta Canada T2N 1N4 